\title{
Correlation analysis of severe aplastic anemia immunosuppressive therapy and human leukocyte antigen alleles in pediatric patients
}

\author{
RU-TING FU, HONG-MAN XUE, BI-HONG ZHANG, JIAN WANG, SHAO-FEN LIN and CHUN CHEN \\ Department of Pediatrics, Sun Yat-Sen Memorial Hospital, Sun Yat-Sen University, \\ Guangzhou, Guangdong 510000, P.R. China
}

Received August 25, 2014; Accepted August 20, 2015

DOI: $10.3892 /$ etm.2015.2807

\begin{abstract}
The aim of the present study was to investigate the correlation between the efficacy of immunosuppressive therapy (IST) in children with severe aplastic anemia (SAA) and human leukocyte antigen (HLA) alleles. The polymerase chain reaction-sequence based typing high-resolution genotyping method was used to profile the HLA alleles of 115 SAA cases that were treated with rabbit-antithymocyte globulin (r-ATG) + cyclosporine (CsA) immunosuppressive therapy and 222 normal control subjects. The aim was to compare the frequency distribution of HLA alleles among the IST-effective group, the IST-ineffective group and the healthy control group. The results showed that the gene frequencies (GFs) of HLA-B*15:02, B*40:02, B*48:01, DRB1*09:01, C*01:02, C*03:04, DQB1*03:03 and DQB1*06:02 in the IST-effective group were significantly higher compared with those in the healthy control group, with a statistically significant difference. The GFs of HLA-B*15:11, B*38:01, B*39:05, DRB1*15:01, C*01:02 and $C^{*} 08: 22$ in the IST-ineffective group were significantly increased compared with those in the healthy control group, with a statistically significant difference. The gene frequency of HLA-A*29:01 in the IST-effective group was significantly reduced compared with that in the IST-ineffective group, and the difference was statistically significant. In summary, IST efficacy in children with SAA that express the HLA-B*15:02, B*40:02, B*48:01, DRB1*09:01, C*01:02, C*03:04, DQB1*03:03 and DQB1*06:02 alleles may be superior, while the efficacy may be mitigated in children with SAA who express HLA-A*29:01, B*15:11, B*38:01, B*39:05, DRB1*15:01, C*01:02, C*08:22 alleles.
\end{abstract}

Correspondence to: Professor Chun Chen, Department of Pediatrics, Sun Yat-Sen Memorial Hospital, Sun Yat-Sen University, 107 Yan Jiang Xi Road, Guangzhou, Guangdong 510000, P.R. China E-mail: docchunchen@163.com

Key words: severe aplastic anemia, immunosuppressive therapy, human leukocyte antigen alleles, high-resolution

\section{Introduction}

Aplastic anemia (AA) is an organ-specific blood disease, that is closely associated with immunity and is predominantly mediated by T cells. In addition to failure of the bone marrow's blood-producing function, AA exhibits autoimmune disease characteristics, and an immune-mediated pathogenesis is crucially involved in the occurrence of AA (1-5). Allogeneic hematopoietic stem cell transplantation and immunosuppressive therapy (IST) are the two primary treatment methods for severe AA (SAA) (6-8). Stem cell transplantation is the treatment of choice for children with a human leukocyte antigen (HLA)-matched sibling donor (1). However, IST is the first-line treatment choice for patients with SAA that do not have a suitable sibling donor.

HLA is among the most complex immune genetic systems in the human genome, regulating the internal and external immune function of the entire body. To date, HLA has been associated with a variety of autoimmune diseases (9). AA has also been reported to be associated with HLA (10). Previous studies that have investigated the correlation between HLA and AA have observed that certain specific HLA alleles or genes are genetic factors that mediate the immunopathogenesis of AA (11), and are associated with IST efficacy, since Chapuis et al (12) initially proposed the correlation of HLA-DR2 with AA in 1985. Studies have demonstrated that HLA-DRB1*15 (a HLA-DR2 subtype) is among the genotypes that predisposes a child to develop AA (2-4). In addition, it has been shown that HLA-DRB1*15 is associated with the immunosuppressive therapy of children with AA $(11,13,14)$; children who were positive for HLA-DRB $1 * 15$ exhibited a good response to the simple cyclosporine A (CsA) treatment and enhanced immunosuppressive therapy. However, previous studies concerning the association of HLA with AA in different provinces of China have yielded inconsistent results $(15,16)$, and IST-related studies of HLA are limited.

In the present study, the polymerase chain reaction-sequence-based typing (PCR-SBT) high-resolution genotyping method was used to identify the HLA alleles of 115 children with SAA and 222 non-blood-related healthy control children. The aim was to assess and investigate the correlation between IST efficacy in the children with SAA and HLA alleles, potentially enabling the improved prediction of the efficacy of IST in children with AA. 


\section{Materials and methods}

Subject. The study population consisted of 115 children who were diagnosed with SAA and treated with a combination of rabbit-antithymocyte globulin (r-ATG) + CsA between February 2008 and April 2012 in the Sun Yat-Sen Memorial Hospital (Guangzhou, China). The study population included 54 male and 61 female patients, aged 1.3-15.5 years old, with a median age of 5.7 years. SAA was diagnosed on the basis of the Camitta diagnostic criteria (17), and AA was divided into severe and mild types. Patients with other blood diseases, such as paroxysmal nocturnal hemoglobinuria, anemia and myelodysplastic syndrome, were excluded. The follow-up period was set to conclude at March 2013 or in the event of patient mortality, with a median follow-up time of 30 months and a follow-up range of 12-61 months. Simultaneously, 222 non-blood-related healthy blood donors were randomly selected as a control population. This study was conducted in accordance with the Declaration of Helsinki and with the approval of the Ethics Committee of Sun Yat-Sen University. Written informed consent was obtained from the guardians of all participants.

IST and therapeutic effect evaluation. The IST used in this study was a combination therapy of r-ATG + CsA + methyl prednisolone. r-ATG was purchased from Genzyme Polyclonals SAS (Lyon, France), and was administered at a dose of $3.5 \mathrm{mg} / \mathrm{kg} /$ day dissolved in $250-500 \mathrm{ml}$ saline via 12-18 h continuous intravenous infusion for 5 consecutive days. Methylprednisolone was purchased from Pharmacia \& Upjohn Co., Ltd., (Shanghai, China) and was administered via intravenous infusion, simultaneously with r-ATG, at a dosage of $1-2 \mathrm{mg} / \mathrm{kg} /$ day. On day 5 of r-ATG application, methylprednisolone tablets (Pharmacia \& Upjohn Co., Ltd.) were orally administered for 9 successive days, at a dosage of $1 \mathrm{mg} / \mathrm{kg} /$ day. After this, the dosage was reduced and discontinued within 5 days to prevent serum sickness. CsA from Hangzhou Zhongmei Huadong Pharmaceutical Co. (Hangzhou, China) was administered at a dose of $5-8 \mathrm{mg} / \mathrm{kg} / \mathrm{day}$, as two oral administrations, with a 12 -h interval. CsA administration started on day 14 and continued for $\geq 6$ consecutive months. The dose was adjusted according to the concentration of CsA in the blood (target trough concentration in whole blood of $150-250 \mathrm{ng} / \mathrm{ml}$ at the start of treatment) and the patient's serum creatinine, transaminase and bilirubin levels. In addition, the patients were treated with hematopoietic growth factor (HGF) at a dose of $5 \mu \mathrm{g} / \mathrm{kg} / \mathrm{day}$, and this treatment was withdrawn when the absolute neutrophil count (ANC) reached 1.5 $10^{9} / 1$.

The effects of the intervention were assessed following 6 months of treatment. Treatment efficacy against SAA was assessed using the standards developed at the 1998 International Santa Margherita Ligure Conference (18), which were as follows: i) Complete response (CR), ANC $>1.5 \times 10^{9} / 1$, hemoglobin $(\mathrm{Hb})>110 \mathrm{~g} / 1$, platelet count $(\mathrm{PLT})>100 \times 10^{9} / 1$; ii) partial response (PR), ANC $>0.5 \times 10^{9} / 1, \mathrm{Hb}>80 \mathrm{~g} / 1$, PLT $>20 \times 10^{9} /$; iii) no response (NR), no improvement in blood routine results after the treatment; and iv) relapse, the peripheral blood cells reached CR or PR, then returned to the SAA level, with or without blood component transfusion. After the treatment, CR and PR were considered to indicate an effec- tive treatment. When determining the treatment efficacy, the administration of HGF was discontinued for $>2$ weeks, and the blood routine levels should remain stable for 4 weeks.

Specimen collection. A 2-ml venous blood sample was extracted from each subject, anticoagulated using ethylenediaminetetraacetic (EDTA) and stored at $-30^{\circ} \mathrm{C}$.

Extraction of DNA template. A QIAamp DNA Mini Kit DNA extraction workstation (Qiagen, Hilden, Germany) was used to extract 3,500 copies of DNA from the blood samples. The optical density of the samples was measured at 260 and $280 \mathrm{~nm}$ to enable the DNA concentrations to be adjusted to a final concentration of $2 \mathrm{ng} / \mathrm{ml}$.

PCR amplification and electrophoresis. The extracted DNA was set as the template, together with $10-\mu 1$ PCR reaction system containing Taq enzyme (0.0375 units) and a primer-containing (1 $\mu \mathrm{mol} / \mathrm{l})$ mixture. These reagents for the amplification were obtained from a PCR-Primer mix which was part of a ROSE HLA-ABDR kit (batch no. SKA000013; ROSE Europe GmbH, Frankfurt am Main, Germany). PCR amplification procedures were as follows: $96^{\circ} \mathrm{C}$ for $120 \mathrm{sec} ; 96^{\circ} \mathrm{C}$ for $30 \mathrm{sec}, 65^{\circ} \mathrm{C}$ for $30 \mathrm{sec}$ and $72^{\circ} \mathrm{C}$ for $120 \mathrm{sec}(5 \mathrm{cycles}) ; 96^{\circ} \mathrm{C}$ for $30 \mathrm{sec}, 62^{\circ} \mathrm{C}$ for $30 \mathrm{sec}$ and $72^{\circ} \mathrm{C}$ for $120 \mathrm{sec}\left(35\right.$ cycles); and finally $10^{\circ} \mathrm{C}$ for $4 \mathrm{~h}$. Following the PCR reaction, $2.5 \mu \mathrm{l}$ mixture was collected for $1.5 \%$ agarose gel electrophoresis, to evaluate the amplification products.

Purification of PCR amplification products. $0.8 \mu 1$ FastAP (1 U/ $\mu 1$; EFO651; Fermentas, Thermo Fisher Scientific, Pittsburgh, PA, USA) and $0.2 \mu 1$ Exonuclease I (20 U/ $\mu 1$; EN0582; Fermentas) were added to the Eppendorf tube of the corresponding positive well. After mixing, the mixture was reacted (Bio-Rad Laboratories, Inc., Hercules, CA, USA) at $37^{\circ} \mathrm{C}$ for $15 \mathrm{~min}$ and $85^{\circ} \mathrm{C}$ for $15 \mathrm{~min}$.

Sequencing reaction. To conduct the sequencing reaction, $5 \mu 1$ specific PCR amplification products were diluted with DNA diluent (Takara Bio, Inc., Otsu, Japan) to a ratio of 1:10. The mixture was then divided into A, T, C and $\mathrm{G}$ tubes for forward and reverse sequencing amplification of each target exon using a 3730xl DNA Analyzer (Applied Biosystems Life Technologies, Foster City, CA, USA). The sequencing reaction conditions were as follows: $96^{\circ} \mathrm{C}$ for $60 \mathrm{sec} ; 96^{\circ} \mathrm{C}$ for $100 \mathrm{sec}$ and $60^{\circ} \mathrm{C}$ for $120 \mathrm{sec}\left(40\right.$ cycles); and finally $10^{\circ} \mathrm{C}$ for $4 \mathrm{~h}$.

Purification of sequencing products. Following the sequencing reaction, $2.5 \mu 1125 \mathrm{mmol} / 1$ EDTA was added to each well. After mixing, $15 \mu \mathrm{l}$ absolute ethanol was added, followed by thorough mixing and the plate was kept in the dark at room temperature for $10 \mathrm{~min}$. The mixture was then subjected to centrifugation at $2,250 \mathrm{x} \mathrm{g}$ for $30 \mathrm{~min}$. The plate was placed upside down on absorbent paper, and centrifuged at $180 \mathrm{x} \mathrm{g}$ for $1 \mathrm{~min}$. After washing with $70 \%$ ethanol, $10 \mu \mathrm{l}$ formamide was added to each well, followed by detection using the $3730 \mathrm{xl}$ DNA Analyzer.

Sequencing electrophoresis. Vertical electrophoresis was conducted with the mixture of purified sequencing products 
Table I. Frequency distributions of HLA-B and DRB1 alleles between the IST-effective group and the healthy control group.

\begin{tabular}{|c|c|c|c|c|c|c|c|c|}
\hline \multirow[b]{2}{*}{ HLA } & \multicolumn{3}{|c|}{ IST-effective group $(n=76)$} & \multicolumn{3}{|c|}{ Healthy control group (n=222) } & \multirow[b]{2}{*}{ P-value } & \multirow[b]{2}{*}{ OR $(95 \% \mathrm{CI})$} \\
\hline & $\mathrm{n}$ & PF & GF & $\mathrm{n}$ & $\mathrm{PF}$ & GF & & \\
\hline B*15:02 & 12 & 0.0789 & 0.0403 & 1 & 0.0023 & 0.0011 & $<0.001$ & $41.438(5.287-324.753)$ \\
\hline$B * 40: 02$ & 7 & 0.0461 & 0.0233 & 3 & 0.0068 & 0.0034 & 0.004 & $7.406(1.864-29.417)$ \\
\hline$B * 48: 01$ & 6 & 0.0395 & 0.0199 & 3 & 0.0068 & 0.0034 & 0.013 & $6.257(1.525-25.675)$ \\
\hline DRB1*09:01 & 34 & 0.2237 & 0.1189 & 58 & 0.1306 & 0.0676 & 0.002 & $2.289(1.331-3.937)$ \\
\hline
\end{tabular}

HLA, human leukocyte antigen; IST, immunosuppressive therapy; PF, phenotype frequency; GF, gene frequency; OR, odds ratio; CI, confidence interval.

from the A, T, C and $\mathrm{G}$ tubes as the sample buffer and placed on the sample rack. The samples were then run using an ABI PRISM 3100 Genetic Analyzer (Applied Biosystems Life Technologies) for automatic sampling. Data Collection 3.0 software, included with the ABI PRISM 3100 Genetic Analyzer, was used to track and collect electrophoresis data over a 6-h period.

Data analysis. Data Analysis for Genomics Software, IMGT HLA Database version 3.7.0 (Beijing Institute of Genomics, China) was used to conduct the data analysis. The nucleotide sequence data were loaded from Worksheet into the Match Tools path. IBU/Hetero values were selected and analysis was conducted in the HLA-Allele database. The Batch Worksheet was then archived, and the preliminary results of nucleotide sequence analysis were obtained. Using the MT Navigator path, the Batch Worksheet was selected, the forward and reverse sequences were edited, and following the sequence consistency correction, output files were saved. Then, using the Match Tools path, the amended Worksheet and the corresponding allele library were selected. Following the manual correction, high-resolution HLA allele typing results could be obtained.

Statistical processing. The antigen phenotype frequency (PF) was the ratio or percentage of a certain antigenic phenotype in the whole population, and the gene frequency (GF) was calculated using the following formula: $1-\sqrt{ } 1-P F$. The difference analysis of GF among the three groups used the multiple-set binary data Pearson $\chi^{2}$ test, in which $\mathrm{P}<0.05$ was considered to indicate a statistically significant difference. The intergroup GF difference analysis used the Pearson $\chi^{2}$ test or continuous corrected $\chi^{2}$ test or Fisher's exact test, with $\mathrm{P}<0.05 / 3$ considered to indicate statistical significance. SPSS software, version 17.0 (SPSS, Inc., Chicago, IL, USA) was used to determine the $\chi^{2}$ value, odds ratio (OR) and $95 \%$ confidence interval $(\mathrm{CI})$. An OR of $>1$ represented a positive correlation, while an $\mathrm{OR}<1$ represented a negative correlation.

\section{Results}

Overall effects. Following the 6-month IST treatment period, treatment was effective in $76 / 115$ cases, corresponding to a treatment response of $66.1 \%$. Among the 76 effective cases, a PR was achieved in 61 cases and a CR was achieved in 15 cases.

Correlation of IST reactivity and HLA alleles. Statistically significant differences were detected in the GFs of HLA-A*29:01, B*13:01, B*15:11, B*15:19, B*35:01, B*38:01, $\mathrm{B} * 39: 03, \mathrm{~B} * 39: 05, \mathrm{~B} * 40: 02, \mathrm{~B} * 44: 02, \mathrm{~B} * 48: 01, \mathrm{~B} * 81: 02$, C*01:02, C*03:02, C*03:04, C*03:17, C*08:22, DRB1*03:01, DRB1*03:08, DRB1*08:09, DRB1*09:01, DRB1*13:01, DRB1*14:54, DRB1*15:01, DQB1*03:03 and DQB1*06:02 among the IST-effective, IST-ineffective and healthy control groups. By contrast, no statistically significant difference was detected in HLA-B*54:01, DRB1*15, DRB1*15:02, DRB1*04, DRB1*07 and DRB1*13:01 among the three groups.

HLA allele frequency distribution. The GFs of HLA-B*15:02 (0.0403 vs. 0.0011), B*13:01 (0.0681 vs. 0.0402), B*40:02 (0.0233 vs. 0.0034$), \mathrm{B} * 48: 01$ (0.0199 vs. 0.0034), DRB1*09:01 $(0.1189$ vs. 0.0676$)$ and DRB1*15:02 (0.0335 vs. 0.0286$)$ in the IST-effective group were significantly higher compared with those in the healthy control group, and the differences were statistically significant (Table I). The GFs of HLA-C*01:02 (0.1022 vs. 0.0320), C*03:04 (0.1194 vs. 0.0616), DQB1*03:03 $(0.1252$ vs. 0.0700$)$ and DQB1*06:02 (0.0470 vs. 0.0102$)$ in the IST-effective group were significantly increased compared with those in the healthy control group, and the differences were statistically significant (Table II), which indicates that children with SAA that express HLA-B*15:02, B*40:02, B*48:01, C*03:04, C*01:02, DRB1*09:01, HLA-B*13:01, DQB1*03:03 and DQB1*06:02 may exhibit an improved response to IST.

The GFs of HLA-B*15:11 (0.0194 vs. 0.0011), B*38:01 (0.0527 vs. 0.0056), B*39:05 (0.0194 vs. 0.0011) and DRB1*15:01 (0.1013 vs. 0.0414) in the IST-ineffective group were significantly elevated compared with those in the healthy control group, and the differences were statistically significant (Table III). The GFs of HLA-C*01:02 (0.0839 vs. 0.0320) and $C^{*} 08: 22(0.0180$ vs. 0.0000$)$ in the IST-ineffective group were significantly increased compared with those in the healthy control group, and the differences were statistically significant (Table IV), indicating that children with SAA that express HLA-B*15:11, B*38:01, B*39:05, C*01:02, C*08:22 and DRB1*15:01 may exhibit a diminished response to IST. 
Table II. Frequency distributions of HLA-C and DQB1 alleles between the IST-effective and healthy control groups.

\begin{tabular}{|c|c|c|c|c|c|c|c|c|}
\hline \multirow[b]{2}{*}{ HLA } & \multicolumn{3}{|c|}{ IST-effective group $(n=49)$} & \multicolumn{3}{|c|}{ Healthy control group $(n=222)$} & \multirow[b]{2}{*}{ P-value } & \multirow[b]{2}{*}{ OR $(95 \% \mathrm{CI})$} \\
\hline & $\mathrm{n}$ & PF & GF & $\mathrm{n}$ & $\mathrm{PF}$ & GF & & \\
\hline C*01:02 & 19 & 0.1939 & 0.1022 & 28 & 0.0631 & 0.0320 & $<0.001$ & $4.388(2.183-8.819)$ \\
\hline C*03:04 & 22 & 0.2245 & 0.1194 & 53 & 0.1194 & 0.0616 & 0.003 & $2.598(1.367-4.937)$ \\
\hline DQB1*03:03 & 23 & 0.2347 & 0.1252 & 60 & 0.1351 & 0.0700 & 0.006 & $2.388(1.266-4.505)$ \\
\hline DQB1*06:02 & 9 & 0.0918 & 0.0470 & 9 & 0.0203 & 0.0102 & $<0.001$ & $5.325(1.991-14.241)$ \\
\hline
\end{tabular}

HLA, human leukocyte antigen; IST, immunosuppressive therapy; PF, phenotype frequency; GF, gene frequency; OR, odds ratio; CI, confidence interval.

Table III. Frequency distributions of HLA-A, B and DRB1 alleles between the IST-ineffective and healthy control groups.

\begin{tabular}{|c|c|c|c|c|c|c|c|c|}
\hline \multirow[b]{2}{*}{ HLA } & \multicolumn{3}{|c|}{ IST-ineffective group $(n=49)$} & \multicolumn{3}{|c|}{ Healthy control group $(n=222)$} & \multirow[b]{2}{*}{ P-value } & \multirow[b]{2}{*}{ OR $(95 \% \mathrm{CI})$} \\
\hline & $\mathrm{n}$ & $\mathrm{PF}$ & GF & $\mathrm{n}$ & $\mathrm{PF}$ & GF & & \\
\hline$B * 15: 11$ & 3 & 0.0385 & 0.0194 & 1 & 0.0023 & 0.0011 & 0.007 & $18.417(1.864-181.940)$ \\
\hline B*38:01 & 8 & 0.1026 & 0.0527 & 5 & 0.0113 & 0.0056 & $<0.001$ & $11.200(3.445-36.413)$ \\
\hline$B * 39: 05$ & 3 & 0.0385 & 0.0194 & 1 & 0.0023 & 0.0011 & 0.007 & $18.417(1.864-181.940)$ \\
\hline DRB 1*03:01 & 0 & 0.0000 & 0.0000 & 29 & 0.0653 & 0.0332 & 0.011 & 0 \\
\hline DRB $1 * 15: 01$ & 15 & 0.1923 & 0.1013 & 36 & 0.0811 & 0.0414 & 0.001 & $3.229(1.545-6.749)$ \\
\hline
\end{tabular}

HLA, human leukocyte antigen; IST, immunosuppressive therapy; PF, phenotype frequency; GF, gene frequency; OR, odds ratio; CI, confidence interval.

Table IV. Frequency distributions of HLA-C alleles between the IST-ineffective and healthy control groups.

\begin{tabular}{|c|c|c|c|c|c|c|c|c|}
\hline \multirow[b]{2}{*}{ HLA } & \multicolumn{3}{|c|}{ IST-ineffective group $(n=28)$} & \multicolumn{3}{|c|}{ Healthy control group $(n=222)$} & \multirow[b]{2}{*}{ P-value } & \multirow[b]{2}{*}{ OR (95\%CI) } \\
\hline & $\mathrm{n}$ & $\mathrm{PF}$ & GF & $\mathrm{n}$ & $\mathrm{PF}$ & GF & & \\
\hline C*01:02 & 9 & 0.1607 & 0.0839 & 28 & 0.0631 & 0.0320 & 0.006 & $3.282(1.352-7.965)$ \\
\hline C*03:02 & 0 & 0.0000 & 0.0000 & 49 & 0.1104 & 0.0568 & 0.002 & 0 \\
\hline C*08:22 & 2 & 0.0357 & 0.0180 & 0 & 0.0000 & 0.0000 & 0.012 & $\infty$ \\
\hline
\end{tabular}

HLA, human leukocyte antigen; IST, immunosuppressive therapy; PF, phenotype frequency; GF, gene frequency; OR, odds ratio; CI, confidence interval.

The GF of HLA-A*29:01 in the IST-effective group was reduced compared with that in the IST-ineffective group (0.0000 vs. 0.0260 ; Table V) and healthy control group, and the difference was statistically significant, indicating that children with SAA that express HLA-A*29:01 may experience reduced therapeutic effects following IST.

\section{Discussion}

Large-sample epidemiological and family studies have indicated that numerous autoimmune diseases, rheumatoid diseases, endocrine diseases, cancers and infectious diseases, among others, are closely associated with HLA (9). This may be due to the effects of certain immune response genes or disease-susceptibility genes, which are HLA-linked and in linkage disequilibrium. Furthermore, a large number of studies have identified significant associations among different HLA loci and various blood diseases (13). Peng et al (19) reported that the TNF- $\alpha$ allele on the HLA-DRB1 locus was highly expressed in patients with AA, altering the secondary structure of DNA via cis-acting elements. This modification affects TNF- $\alpha$ gene transcription and protein synthesis, resulting in the upregulation of TNF- $\alpha$ expression by $20-40 \%$. These results suggest that certain specific HLA genotypes are genetic indicators of an increased susceptibility to AA immunopathogenesis. The results of previous studies concerning the association between HLA alleles and AA susceptibility are summarized in Table VI (10,11,13,15,16,20-23). 
Table V. HLA-A allele frequency distribution in the IST-effective and the IST-ineffective groups.

\begin{tabular}{llcccccr}
\hline & \multicolumn{3}{c}{ IST-effective group $(\mathrm{n}=76)$} & & \multicolumn{3}{c}{ IST-ineffective group $(\mathrm{n}=39)$} \\
\cline { 2 - 7 } HLA-A & $\mathrm{n}$ & $\mathrm{PF}$ & $\mathrm{GF}$ & $\mathrm{n}$ & $\mathrm{PF}$ & GF \\
\hline A*29:01 & 0 & 0.0000 & 0.0000 & 4 & 0.0513 & 0.0260 \\
\hline
\end{tabular}

HLA, human leukocyte antigen; IST, immunosuppressive therapy; PF, phenotype frequency; GF, gene frequency; OR, odds ratio; CI, confidence interval.

Table VI. Reports of HLA alleles and AA susceptibility in different countries/regions.

\begin{tabular}{|c|c|c|c|c|}
\hline HLA allele & Country/region & $\mathrm{AA}, \mathrm{n}$ & $\mathrm{OR} / \mathrm{RR}$ & Year (Ref.) \\
\hline$B * 48: 01$ & Chinese Han & 80 & 12.000 & $2012(12)$ \\
\hline \multirow[t]{2}{*}{ DRB $1 * 15$} & North American caucasian & $59^{a}$ & 2.72 & $2002(13)$ \\
\hline & Pakistan & 61 & 1.97 & 2009 (14) \\
\hline \multirow[t]{5}{*}{ DRB $1 * 15: 01$} & Northern Chinese Han & 102 & $3.07^{\mathrm{b}}$ & $2000(15)$ \\
\hline & Russia & $44^{\mathrm{a}}$ & 2.9 & $2001(16)$ \\
\hline & Japan & 140 & 3.43 & 2007 (17) \\
\hline & Korea & 109 & 2.74 & 2008 (18) \\
\hline & Korea & 37 & 2.9 & 2010 (19) \\
\hline $\mathrm{DRB} 1 * 15: 02$ & Japan & 140 & 2.39 & 2007 (17) \\
\hline DRB $1 * 04$ & Russia & 44 & 2.4 & $2001(16)$ \\
\hline $\mathrm{DRB} 1 * 07$ & Iran & 35 & - & $2008(20)$ \\
\hline DRB1*09:01 & Chinese Han & 96 & 3.076 & $2012(12)$ \\
\hline DRB 1*13:01 & Chinese Han & 96 & - & $2012(12)$ \\
\hline
\end{tabular}

${ }^{\mathrm{a} S A A}$; ${ }^{\mathrm{B} R}$ value, all other values in the column are OR values. HLA, human leukocyte antigen; AA, aplastic anemia; OR, odds ratio; RR, relative risk.

In the present study, the prevalence of the B*13:01 allele exhibited a statistically significant difference among the three groups. In the comparison between the IST-effective and healthy control groups, the GF of $\mathrm{B}^{*} 13: 01$ was higher in the IST-effective group (0.0681 vs. 0.0402), although the difference was not statistically significant. $B * 54: 01$ prevalence did not differ among the three groups. $\mathrm{B} * 48: 01$ showed a statistically significant difference among the three groups, and in the comparison between the IST-effective and healthy control group, the GF of $\mathrm{B}^{* 48: 01}$ was higher in the IST-effective group (0.0199 vs. 0.0034$)$, and the difference was statistically significant, suggesting that children with AA that possess the $B^{*} 48: 01$ susceptibility gene may experience improved IST efficacy. DRB1*09:01, DRB1*15:01 and DRB1*13:01 exhibited a statistically significant difference among the three groups. The GF of DRB1*09:01 was significantly increased in the IST-effective group compared with the control group (0.1189 vs. 0.0676$)$, suggesting that children with AA that possess the DRB1*09:01 susceptibility gene may exhibit an improved response to IST. The GF of DRB1*15:01 was significantly higher in the IST-ineffective group compared with the control group (0.1013 vs. 0.0414), suggesting that children with AA that possess the DRB1*15:01 susceptibility gene may exhibit a reduced response to IST. No difference was detected in DRB1*15, DRB1*15:02, DBR1*04, DRB1*07 and DRB1*13:01 among the three groups.

The results of previous studies concerning the correlation between IST efficacy in children with AA and HLA alleles are shown in Table VII. The present study analyzed the disease status of 115 children with SAA, after 6 months of IST treatment. Among the 115 cases, treatment was classed as effective in 76 cases, while 39 cases were ineffective, resulting in a 6-month efficiency rate of $66.1 \%(76 / 115)$. Furthermore, PCR-SBT high-resolution genotyping was used to identify the HLA alleles among these 115 SAA cases, in addition to 222 non-blood-related healthy donors, in order to compare GFs among the IST-effective, IST-ineffective and healthy control groups. The results demonstrate that the GFs of HLA-B*15:02, B*40:02, B*48:01, C*01:02, C*03:04, DRB1*09:01, DQB1*03:03 and DQB1*06:02 were significantly higher in the IST effective group compared with the healthy control group, and the differences were statistically significant. The GFs of HLA-A*29:01, B*15:11, B*38:01, B*39:05, $C^{*} 01: 02, C^{*} 08: 22$ and DRB1*15:01 were significantly higher in the IST-ineffective group compared with the healthy control group, and the differences were statistically significant. The 
Table VII. Reports of correlation between HLA alleles and IST efficacy in AA patients from different countries/regions.

\begin{tabular}{|c|c|c|c|c|c|}
\hline HLA alleles & Country/region & $\mathrm{AA}(\mathrm{n})$ & Treatment & OR & Year (Ref.) \\
\hline \multirow[t]{9}{*}{ DRB $1 * 15: 01$} & Japan & 20 & CsA & - & $1992(21)$ \\
\hline & Japan & 59 & CsA & - & $1994(22)$ \\
\hline & Japan & 52 & $\mathrm{CsA}+\mathrm{ATG}$ & - & $1996(23)$ \\
\hline & Turkey & 40 & $\mathrm{CsA} ; \mathrm{CsA} \pm \mathrm{ATG} / \mathrm{ALG}$ & - & $1997(24)$ \\
\hline & Turkey & 33 & $\mathrm{CSA}+\mathrm{ATG}+\mathrm{HDMP}$ & 5.25 & $2002(25)$ \\
\hline & Japan & 140 & $\mathrm{ATG}+\mathrm{CsA}$ & - & 2007 (17) \\
\hline & Korea & $37^{\mathrm{a}}$ & $\mathrm{ATG} / \mathrm{ALG}+\mathrm{CsA}$ & 26.3 & 2010 (19) \\
\hline & Chinese Han & 80 & $\mathrm{ATG}+\mathrm{CsA}$ & $3.272^{\mathrm{c}}$ & $2012(12)$ \\
\hline & Chinese Han & 80 & $\mathrm{ATG}+\mathrm{CsA}$ & $0.127^{\mathrm{d}}$ & $2012(12)$ \\
\hline DRB $1 * 04$ & Russia & $44^{\mathrm{a}}$ & ALG/CsA; ALG; CsA; HSCT; MT & 6.7 & $2001(16)$ \\
\hline DRB $1 * 04: 05$ & Korea & $37^{\mathrm{a}}$ & $\mathrm{ATG} / \mathrm{ALG}+\mathrm{CsA}$ & - & 2010 (19) \\
\hline DRB 1*09:01 & Chinese Han & 80 & $\mathrm{ATG}+\mathrm{CsA}$ & $4.833^{\mathrm{b}}$ & $2012(12)$ \\
\hline DQB1*04:01 & Korea & $37^{\mathrm{a}}$ & ATG/ALG + CsA & - & $2010(19)$ \\
\hline DQB1*06:02 & Korea & $37^{\mathrm{a}}$ & ATG/ALG + CsA & 45.5 & 2010 (19) \\
\hline
\end{tabular}

${ }^{\text {aSAA; }}{ }^{\mathrm{b} I S T}$ effective group vs. control group; ${ }^{\mathrm{c} I S T}$ ineffective group vs. control group; ${ }^{\mathrm{d} I S T}$ effective group vs. IST-ineffective group. HLA, human leukocyte antigen; IST, immunosuppressive therapy; AA, aplastic anemia; OR, odds ratio; CsA, cyclosporine; ATG, antithymocyte globulin; ALG, antilymphocyte globulin; HSCT, hematopoietic stem cell transplantation; MT, marrow transplantation; HDMP, high dose methyl prednisolone.

GF of HLA-A*29:01 in the IST-effective was significantly reduced compared with that in the IST-ineffective group, and the difference was statistically significant.

HLA-DR15, in combination with simple CsA treatment and enhanced IST, has been found to exhibit good therapeutic effects, while DR4 (of which DRB1*04 is a subtype) is associated with poor therapeutic effects. The correlation analysis of HLA-DR2 and its subgroups with IST effects has yielded a number of conflicting results, due to the different IST treatments used $(14,24-27)$. It is generally considered that DR2 and its subgroups are associated with the effectiveness of pure CsA treatment or enhanced IST, but irrelevant for simple ATG or antilymphocyte globulin (ALG) treatment. Among Japanese AA patients, the reactivity of pure CsA treatment or enhanced IST in DR2 $2^{+}$or DRB1*15:01+ patients was significantly higher than that in DR2 ${ }^{-}$or DRB1*15:01' patients $(22,24-26)$. However, no difference in the reactivity of pure ATG treatment was observed between these kinds of patients (20). Among Turkish patients, IST reactivity in DR2 ${ }^{+}$patients was significantly higher compared with that in DR2- patients $(14,27)$; this difference was only expressed in patients receiving treatment with CsA alone or with a combination of CsA + ATG/ALG (27).

In the present study, the GF of DRB1*15:01 in the IST-ineffective group was significantly higher compared with that in the healthy control group $(\mathrm{P}=0.001, \mathrm{OR}=3.229)$. This difference was statistically significant, suggesting that children with SAA that express DRB1*15:01 may exhibit a poor therapeutic response to enhanced combined IST. The GF of DRB1*15:02 in the IST-effective group was higher compared with that in the IST ineffective group (0.0335 vs. 0.0194); however, no significant difference was detected between these two groups. As HLA exhibits racial and regional differences, and the methods and doses of IST used in different regions may vary, the correlation of DRB1*15:01 and DRB1*15:02 with IST efficacy requires investigation in further studies with an increased sample size, and in different populations from various regions.

A previous study observed that among the HLA-II alleles, DR4 was particularly associated with the poor IST efficacy. Among the Russian patients with AA that received CsA treatment, DR4 was associated with poor IST efficacy (21). However, Song et al detected no association between DR4 or HLA-DR4-Ala74 $\beta$ and AA susceptibility (10). Furthermore, Song et al (11) investigated the association between IST efficacy and HLA-II in 37 cases of SAA in Korean patients, and the results suggested that although DR4 was not associated with AA susceptibility, DR4 was associated with a poor response to IST. In particular, the IST reactivities in DRB1*04:05+, DR4-Ala74 $\beta^{+}$and HLA-DR4 ${ }^{+}$patients were significantly reduced compared with those of DR4- patients (5.9 vs. 60.0\%). In addition, Nakao et al observed that among the patients with AA that received CsA therapy only, DR4 was associated with a poor IST response (24). The target analysis of 13 children with AA detected no significant correlation between DR4 and treatment efficacy. In the present study, among the 115 children with SAA, the GFs of DR4 in the three groups showed no significant difference. However, the correlation between HLA-DR4 and SAA efficacy requires further investigation in future studies with an expanded sample size.

The mechanism underlying the association between HLA-DR alleles and IST response remains unclear. Certain specific HLA alleles may be associated with the pathogenesis of AA; therefore, T cells may be mediating and damaging hematopoietic stem cells/progenitor cells. Patients that express certain specific HLA alleles may exhibit an improved IST response. HLA-DR2 (DR15) is an example of this; the overexpression 
of DR2 in patients with AA correlates with good IST efficacy. Although HLA-DR4 was not overexpressed in the patients with AA, DR4 may be associated with immune-mediated AA pathogenesis. In a previous study, clones of T cells, which were isolated from DRB1*04:05 and *15:01-expressing AA patients, revealed that HLA-DRB1*04:05 is able to restrict the cytotoxicity of autologous and allogeneic hematopoietic stem cells, indicating that this type of cytotoxic T cell may serve a function in the pathogenesis of AA (27).

In summary, the correlation between IST efficacy in children with SAA and HLA alleles remains unclear. The efficacy of IST, as the first-line treatment for non-HLA-matched sibling-donor SAA patients, may be affected by numerous factors. Further studies are required to investigate IST efficacy-associated HLA alleles, and enable additional IST efficacy-associated HLA alleles to be identified. The results of the present study support the further investigation of IST efficacy-associated HLA alleles, with the aim of improving the efficacy of IST in children with SAA.

\section{Acknowledgements}

This study was supported by National Nature Scientific Grant of China (no. 81370625) and Nature Scientific Grant of Guangdong Province (no. S2011010002648) and International Cooperation Grant of Guangdong Province (no. 2011B050400023).

\section{References}

1. Brodsky RA and Jones RJ: Aplastic anaemia. Lancet 365: 1647-1656, 2005.

2. Young NS, Calado RT and Scheinberg P: Current concepts in the pathophysiology and treatment of aplastic anemia. Blood 108: 2509-2519, 2006.

3. Risitano AM and Perna F: Aplastic anemia: Immunosuppressive therapy in 2010. Pediatr Rep 3: e7, 2011.

4. Dolberg OJ and Levy Y: Idiopathic aplastic anemia: Diagnosis and classification. Autoimmun Rev 13: 569-573, 2014.

5. Hartung HD, Olson TS and Bessler M: Acquired aplastic anemia in children. Pediatr Clin North Am 60: 1311-1336, 2013.

6. Scheinberg P and Young NS: How I treat acquired aplastic anemia. Blood 120: 1185-1196, 2012.

7. Guinan EC: Diagnosis and management of aplastic anemia Hematology Am Soc Hematol Educ Program 2011: 76-81, 2011.

8. Korthof ET, Békássy AN and Hussein AA: Management of acquired aplastic anemia in children. Bone Marrow Transplant 48 : 191-195, 2013.

9. Miyadera $\mathrm{H}$ and Tokunaga $\mathrm{K}$ : Associations of human leukocyte antigens with autoimmune diseases: Challenges in identifying the mechanism. J Hum Genet: Aug 20, 2015 (Epub ahead of print).

10. Song EY, Park S, Lee DS, Cho HI and Park MH: Association of human leukocyte antigen-DRB1 alleles with disease susceptibility and severity of aplastic anemia in Korean patients. Hum Immunol 69: 354-359, 2008
11. Song EY, Kang HJ, Shin HY, Ahn HS, Kim I, Yoon SS, Park S, Kim BK and Park MH: Association of human leukocyte antigen class II alleles with response to immunosuppressive therapy in Korean aplastic anemia patients. Hum Immunol 71: 88-92, 2010.

12. Chapuis B, Von Fliedner VE, Jeannet M, Merica H, Vuagnat $P$, Gratwohl A, Nissen C and Speck B: Increased frequency of DR2 in patients with aplastic anaemia and increased DR sharing in their parents. Br J Haematol 63: 51-57, 1986.

13. Saunthararajah Y, Nakamura R, Nam JM, Robyn J, Loberiza F, Maciejewski JP, Simonis T, Molldrem J, Young NS and Barrett AJ: HLA-DR15 (DR2) is overrepresented in myelodysplastic syndrome and aplastic anemia and predicts a response to immunosuppression in myelodysplastic syndrome. Blood 100: 1570-1574, 2002.

14. Oguz FS, Yalman N, Diler AS, Oguz R, Anak S and Dorak MT: HLA-DRB1*15 and pediatric aplastic anemia. Haematologica 87: 772-774, 2002.

15. Chen C, Lu S, Luo M, Zhang B and Xiao L: Correlations between HLA-A, HLA-B and HLA-DRB1 allele polymorphisms and childhood susceptibility to acquired aplastic anemia. Acta Haematol 128: 23-27,2012.

16. Shao W, Tian D, Liu C, Sun X and Zhang X: Aplastic anemia is associated with HLA-DRB1*1501 in northern Han Chinese. Int J Hematol 71: 350-352, 2000.

17. Camitta BM, Thomas ED, Nathan DG, et al: Severe aplastic anemia: A prospective study of the effect of early marrow transplantation on acute mortality. Blood 48: 63-70, 1976.

18. International Meeting on Antimicrobial Chemotherapy in Clinical Practice (ACCP). Santa Margherita Ligure, Italy. October 27-30, 1999. Proceedings. Int J Antimicrob Agents 16: 257-378, 2000.

19. Peng J, Liu C, Zhu K, Zhu Y, Yu Y, Li J, Hou M, Chen X, Xu C and Zhang M: The TNF2 allele is a risk factor to severe aplastic anemia independent of HLA-DR. Hum Immunol 64: 896-901, 2003.

20. Rehman S, Saba N, Khalilullah, Munir S, Ahmed P and Mehmood T: The frequency of HLA class I and II alleles in Pakistani patients with aplastic anemia. Immunol Invest 38: 812-819, 2009.

21. Kapustin SI, Popova TI, Lyshchov AA, Myanitov EN, Blinov MN and Abdulkadyrov KM: HLA-DR4-Ala74 beta is associated with risk and poor outcome of severe aplastic anemia. Ann Hematol 80: 66-71, 2001.

22. Sugimori C, Yamazaki H, Feng X, Mochizuki K, Kondo Y, Takami A, Chuhjo T, Kimura A, Teramura M, Mizoguchi H, et al: Roles of DRB1*1501 and DRB1*1502 in the pathogenesis of aplastic anemia. Exp Hematol 35: 13-20, 2007.

23. Yari F, Sobhani M, Vaziri MZ, Bagheri N, Sabaghi F and Talebian A: Association of aplastic anaemia and Fanconi's disease with HLA-DRB1 alleles. Int J Immunogenet 35: 453-456, 2008.

24. Nakao S, Yamaguchi M, Saito M, Yasue S, Shiobara S, Matsuda T, Nitta M and Sasaki M: HLA-DR2 predicts a favorable response to cyclosporine therapy in patients with bone marrow failure. Am J Hematol 40: 239-240, 1992.

25. Nakao S, Takamatsu H, Chuhjo T, Ueda M, Shiobara S, Matsuda T, Kaneshige T and Mizoguchi H: Identification of a specific HLA class II haplotype strongly associated with susceptibility to cyclosporine-dependent aplastic anemia. Blood 84: 4257-4261, 1994

26. Nakao S, Takami A, Sugimori N, Ueda M, Shiobara S, Matsuda T and Mizoguchi H: Response to immunosuppressive therapy and an HLA-DRB1 allele in patients with aplastic anaemia: HLA-DRB1*1501 does not predict response to antithymocyte globulin. Br J Haematol 92: 155-158, 1996.

27. Ihan O, Beksaç M, Arslan O, Ozcan M, Koç H, Akan H, Gürman G, Konuk N and Uysal A: HLA DR2: A predictive marker in response to cyclosporine therapy in aplastic anemia. Int J Hematol 66: 291-295, 1997. 